\title{
Artifact Trapping During Time Reversal Photoacoustic Imaging for Acoustically Heterogeneous Media
}

\author{
Benjamin T. Cox* and Bradley E. Treeby
}

\begin{abstract}
Several different reconstruction algorithms have been proposed for photoacoustic tomography, most of which presuppose that the acoustic properties of the medium are constant and homogeneous. In practice, there are often unknown spatial variations in the acoustic properties, and these algorithms give, at best, only approximate estimates of the true image. The question as to which approach is the most robust in these circumstances is therefore one of practical importance. Image reconstruction by "time reversal"- using a numerical propagation model with a time-varying boundary condition corresponding to the measured data in reversed temporal order-has been shown to be less restrictive in its assumptions than most, and therefore a good candidate for a general and practically useful algorithm. Here, it is shown that such reconstruction algorithms can "trap" time reversed scattered waves, leading to artifacts within the image region. Two ways to mitigate this effect are proposed.
\end{abstract}

Index Terms-Artifact trapping, image reconstruction, photoacoustic imaging, time reversal.

\section{INTRODUCTION}

$\mathbf{P}$ HOTOACOUSTIC tomography (PAT) is an emerging technique for imaging regions of optical absorption in light-scattering media, such as soft biological tissue. PAT images are related to the optical absorption coefficient, and so carry structural, compositional, and functional information about the tissue. PAT has been widely demonstrated, and is increasingly being used in the biomedical and life sciences (for reviews, see [1] and [2], and references therein). It has passed the "proof-of-principle" phase, and the focus is now turning to refining its capabilities for use as a practical, high resolution, experimental imaging technique. The enhancements necessary to achieve this aim may be effected through improvements in the image reconstruction process, as well as through the development of better hardware, such as more sensitive and smaller detector elements. Progress in image reconstruction

Manuscript received August 28, 2009; accepted September 06, 2009. First published November 03, 2009; current version published February 03, 2010. This work was supported by the Engineering and Physical Sciences Research Council, U.K. Asterisk indicates corresponding author.

*B. T. Cox is with the Department of Medical Physics and Bioengineering, University College, WC1E 6DE London, U.K. (e-mail: bencox@mpb.ucl.ac. uk).

B. E. Treeby is with the Department of Medical Physics and Bioengineering, University College, WC1E 6DE London, U.K. (e-mail: btreeby@mpb.ucl.ac. uk).

Digital Object Identifier 10.1109/TMI.2009.2032358 could be realized either by 1) devising new image reconstruction algorithms that can incorporate additional information that may be known about the tissue (e.g., sound speed distribution, or prior structural details) or 2) finding which algorithms are most robust to the uncertainties that will be found in practice (e.g., in material properties, detector position, etc.). Both 1) and 2) could be achieved by generalizing existing imaging algorithms by lessening their dependence on idealizations and assumptions that are not realistic for typical imaging scenarios.

This paper is concerned with the robustness of image reconstruction using "time reversal." It highlights one way in which the particular nature of time reversal image reconstruction may, in fact, exaggerate the level of image artifacts due to unknown variations in density and sound speed. It is demonstrated that the enforced time reversal boundary condition can "trap" artifacts in the final image, and that by truncating the data, or introducing a thresholded boundary condition, this artifact trapping can be mitigated to some extent.

\section{Photoacoustic Time Reversal Imaging}

\section{A. Photoacoustic Initial Value Problem}

When a pulse of light enters a turbid media with regions of nonzero absorption, the light is scattered until it is either absorbed or exits from the tissue. Under conditions whereby the absorbed optical energy is converted to heat there is a localized increase in temperature and-if the heating occurs much more quickly than the thermal relaxation time of the tissue-a proportional increase in pressure. Because of the elasticity of the tissue, this excess pressure propagates away as an acoustic wave. This is known as the photoacoustic effect, and the excess pressure is known as the initial acoustic pressure distribution. It is this initial pressure distribution that PAT is designed to image. (When longer wavelength electromagnetic radiation, e.g., RF or microwave, is used in place of light, this technique is referred to as thermoacoustic tomography. The acoustic image reconstruction problem, however, is identical in both cases.) When the duration of the light pulse is a few nanoseconds, broadband ultrasonic pulses with a bandwidth of tens of megahertz are emitted. Typically, such light pulses are sufficiently short for the acoustic propagation to be modelled as an initial value problem. As the pressure amplitudes are usually too low for nonlinear effects to be important, the photoacoustic forward problem may be written as

$$
\left[\frac{\partial^{2}}{\partial t^{2}}-c(x)^{2} \rho(x) \nabla \cdot\left(\frac{1}{\rho(x)} \nabla\right)\right] p(x, t)=0
$$




$$
\left.p\right|_{t=0}=p_{0}(x),\left.\quad \frac{\partial p}{\partial t}\right|_{t=0}=0
$$

where $p(x, t)$ is the acoustic pressure at time $t \in \mathbb{R}^{+}$and point $x \in \Omega \subset \mathbb{R}^{n}$ inside the imaging region $\Omega$ ( $n$ is typically 2 or $3), p_{0}(x)$ is the initial distribution of acoustic pressure, and the mass density $\rho(x)$ and sound speed $c(x)$ may vary with position. (The effect of spatial variation in the sound speed on PAT has been studied by a number of authors [3]-[6] but little has been said of the effect of density heterogeneities. Both are included in the models used in this paper.)

\section{B. Image Reconstruction}

The photoacoustic image reconstruction problem is to estimate $p_{0}(x)$ given measurements of $p(x, t)$ on an arbitrary measurement surface $S$, where $p_{s}(t) \equiv p\left(x_{s}, t\right)$ and $x_{s} \in S$. Here the measurement surface $S$ and $\partial \Omega$, the boundary of $\Omega$, are taken to be coincident. Most of the work on photoacoustic image reconstruction has considered only acoustically homogeneous media where $\rho$ and $c$ are constant and (1) is replaced with the wave equation for homogeneous media

$$
\left(\frac{\partial^{2}}{\partial t^{2}}-c^{2} \nabla^{2}\right) p(x, t)=0
$$

Several different PAT image reconstruction formulas that are exact for acoustically homogeneous media have been proposed, even though in practice the medium, e.g., soft tissue, is likely to contain acoustic heterogeneities. However, as typically only the spatially-averaged acoustic properties are known, algorithms based on exact solutions, but using mean values of the material parameters as if they were true values, can be used to obtain good approximations to the true image.

In addition to these "homogeneous-exact" algorithms, a number of approximate algorithms have also been successfully used for PAT (for more details, see [2] and [5]). Given the many reconstruction methods now available, an important question arises: which is the best to use in real imaging scenarios? When looking for the "best" algorithm to use in practice, several factors must be taken into consideration, including accuracy, speed, generality, memory requirements, robustness to uncertainty in the positions of the sensors or partial data, robustness to noise and errors in the measured data, and so on. There is no a priori reason why an "exact-homogeneous" algorithm must perform better than an approximate algorithm when all these criteria are considered. Indeed, when there are unknown acoustic heterogeneities, an exact-homogeneous solution is itself only approximate.

\section{Time Reversal Imaging}

The notion of recreating acoustic pressure fields by retransmitting measured acoustic pressure signals into the medium in reversed temporal order has been used as an experimental technique for some years, and arose by analogy with the concept of phase conjugation in optics [7]. Since then, a great deal of work has been done on experimental demonstrations and simulations of time reversal in acoustics, notably by Fink and colleagues
[8], [9], with applications such as nondestructive evaluation and focussing through scattering media in mind. Bossy et al. [10] have shown experimentally that acoustic waves generated photoacoustically from an optical absorber can be refocused onto the absorber.

The application of the idea of time reversal to tomography was suggested by $\mathrm{Xu}$ and Wang [11], and Burgholzer et al. [12] demonstrated that time reversal of a numerical model (rather than the real, physical retransmission of the signals) could be used as an imaging algorithm for PAT. The technique exploits the fact that the solution, $p(x, t)$, to the initial value problem [(3) and (2) in the homogeneous case] is identical to the solution $\breve{p}(x, \breve{t}=T-t)$ of the boundary value problem given by (3) [or (1) if $\rho(x)$ and $c(x)$ are known] and the conditions

$$
\check{p}(x, \check{t}=0)=0, \quad \check{p}\left(x_{s}, \check{t}\right)=p_{s}(t=T-\check{t})
$$

where the reverse-time variable $\check{t}$ runs from 0 to $T$, and the image appears at $\breve{t}=T$ as $p_{0}(x)=p(x, t=0)=\check{p}(x, \breve{t}=T)$. The time limit $T$ is chosen to be a time after which $p(x, t>$ $T)=0$ in $\Omega$, which is guaranteed in odd dimensions and homogeneous media by Huygens' principle, and is approximately true in even dimensions and heterogeneous media for sufficiently large $T$. It will be helpful to distinguish between the measurement domain $\Omega$, the physical region in which the acoustic waves propagate out from the photoacoustic sources, and the reconstruction domain $\check{\Omega}$ with boundary $\breve{S}$, the numerical space into which waves are propagated during the time reversal image reconstruction. $\check{\Omega}$ and $\breve{S}$ are in silico equivalents to the real world $\Omega$ and $S$. In a practical sense, the reconstruction is performed by using the acoustic pressure time histories measured on $S$ for $t=0$ to $T$ in time reversed order as an enforced (time-varying) Dirichlet boundary condition on $\breve{S}$ within a numerical acoustic propagation model. $\mathrm{Xu}$ and Wang [11] point out that this is equivalent to the re-transmission of the pressure in a reflective cavity formed by $\breve{S}$ where the time reversed $p_{s}(t)$ is injected as a source rather than by imposing a boundary condition.

Recently, time reversal photoacoustic imaging has been described as the "least restrictive" imaging algorithm on the basis that it relies on fewer assumptions than many other image reconstruction algorithms [6], [13]. For example, it is applicable to closed measurement surfaces of any shape, is immune to acoustic sources outside the measurement surface, and makes no assumptions about the initial time rate of change of the pressure (or equivalently the initial acoustic particle velocity). It has also been shown to work reasonably well even when certain assumptions fail, e.g., with a heterogeneous sound speed distribution, and in two dimensions [6], [13]. In addition to these advantages, there is no axiomatic constraint on the efficiency of the algorithm; it is as fast as the numerical propagation code on which it is based. For these reasons, it would seem to be the leading candidate as a generally applicable and robust photoacoustic imaging algorithm. However, it is shown below that the boundary condition inherent in time reversal imaging can act as a reflector to trap the time reversed versions of waves scattered by acoustic heterogeneities, leading to a greater level of artifacts in the image. 
(a) Forward propagation

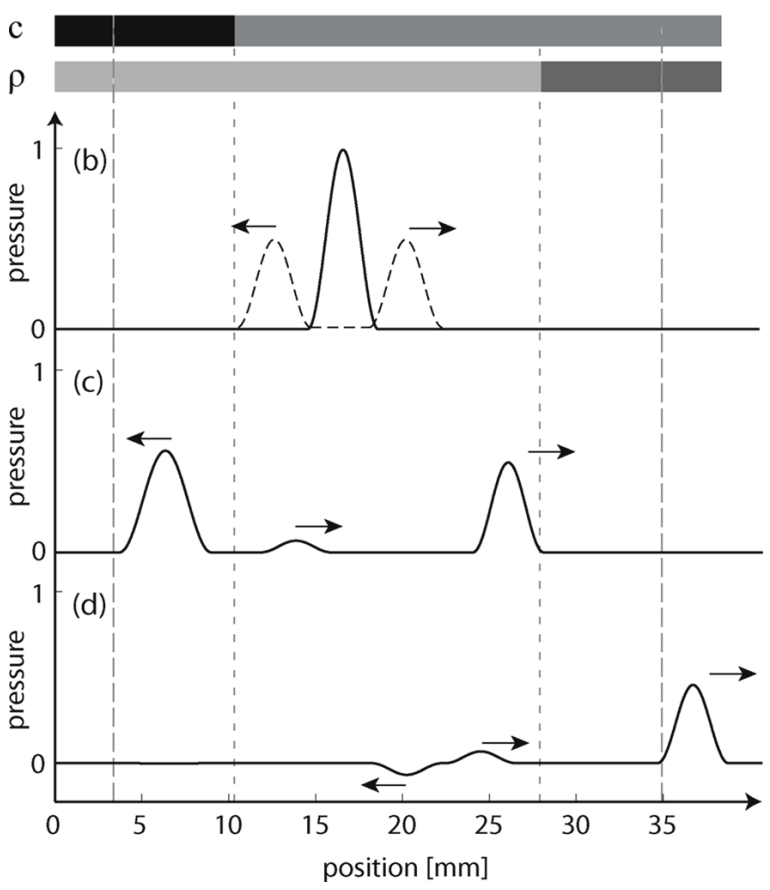

(e)

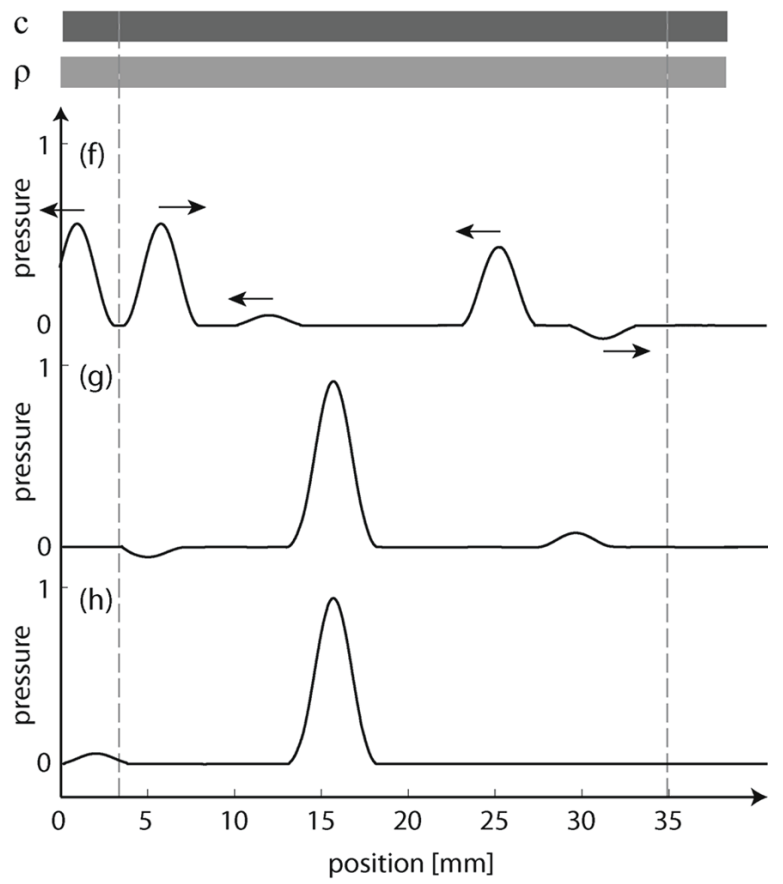

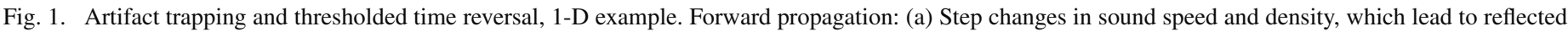

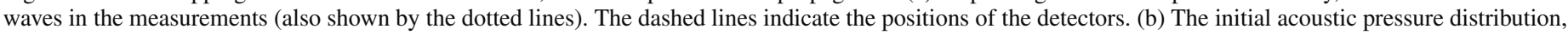

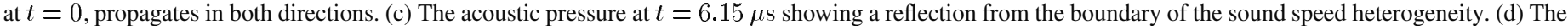

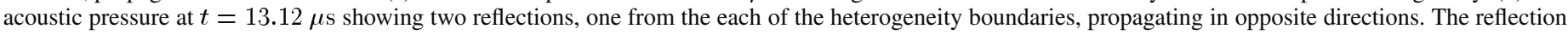

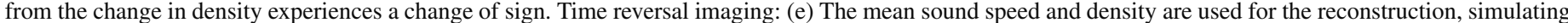

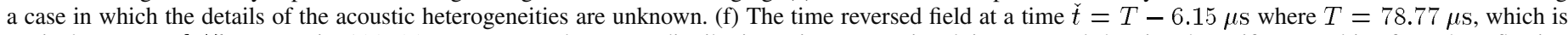

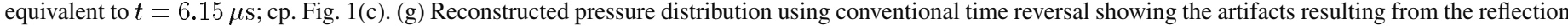

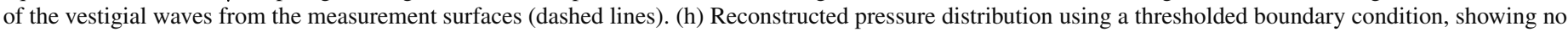
artifacts, as the vestigial waves have propagated across the boundary.

\section{ARTIFACt TRAPPING}

When time reversal image reconstruction is performed in a medium with known acoustic properties, the waves in $\check{\Omega}$ generated by the time-varying boundary condition interfere in such a way as to reproduce exactly, in a time reversed, inward-going sense, the outward-going pressure field that resulted in the boundary measurements. However, if $\rho$ and $c$ vary spatially but only their mean values are known (as is typically the case in practice), the time reversed waves will no longer interfere precisely to reproduce the original wavefield. Instead, they will form an approximation to it, but with additional waves present. These extra waves, generally of lower amplitude than the main wave, are the time reversed relatives of the waves scattered from the acoustic heterogeneities during the forward propagation. They persist in the time reversal due to the mismatch between the actual heterogeneous medium in $\Omega$ (in which the time series are recorded) and the homogeneous approximation to it in $\check{\Omega}$, used in the model. These time reversed vestiges of the scattered waves, the remaining traces of evidence for the presence of acoustic heterogeneities, will be referred to as vestigial waves.

During the time reversal reconstruction, the vestigial waves will continue to propagate through $\check{\Omega}$ until they reach the boundary, $\breve{S}$, on which the Dirichlet boundary condition, (4), is being enforced. Depending on the particular values imposed on the boundary at that moment, they may then be reflected back into $\check{\Omega}$. Vestigial waves are artifact-producing waves, in the sense that any of them remaining in $\check{\Omega}$ at $\check{t}=T$ (corresponding to $t=0$ ) would constitute artifacts in the final image. By this mechanism, the Dirichlet boundary condition on $\breve{S}$ can be said to trap image artifacts within $\check{\Omega}$.

It should be noted that this effect of artifact trapping is a property of the reconstruction algorithm, and should not be confused with ray trapping, which is a property of a medium, specifically one with a sound speed distribution which bends the sound rays in such a way that they never reach the measurement surface. In [6], which discusses the effect of ray-trapping on time reversal imaging in PAT, a nontrapping condition is used to refer to a medium with a sound speed distribution such that it does not trap rays; it does not mean that the images are immune to artifact trapping in the sense described in this paper.

\section{A. One-Dimensional Example}

An illustrative 1-D example of artifact trapping is shown in Fig. 1. The propagation medium contains step changes in both sound speed, $c$, and density, $\rho$, as shown in Fig. 1(a). The values in the center are $c=1500 \mathrm{~m} / \mathrm{s}$ and $\rho=1000 \mathrm{~kg} / \mathrm{m}^{3}$, with a $30 \%$ increase in the sound speed on the left and a $30 \%$ increase in the density on the right. (The rather large changes in the acoustic properties used in this pedagogic example were deliberately chosen to help describe the concept of artifact trapping clearly.) A smoothly varying initial acoustic pressure distribution (which in 1-D will be a plane wave) will begin to propagate in both directions, as indicated in Fig. 1(b), towards the 

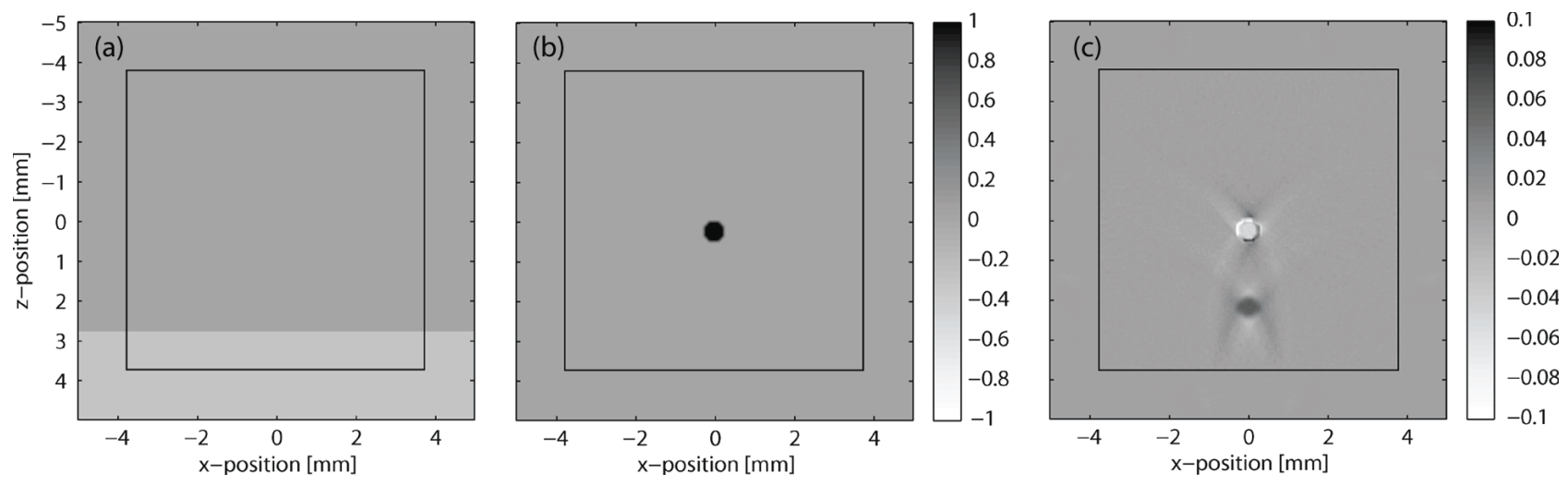

Fig. 2. Artifact trapping, 2-D example: (a) At $z=2.8 \mathrm{~mm}$ there is a step change in the density from $1000 \mathrm{~kg} / \mathrm{m}^{3}$ to $700 \mathrm{~kg} / \mathrm{m}^{3}$. The position of the square measurement surface is shown as a solid black line. (b) The initial pressure distribution is a disc of diameter $0.5 \mathrm{~mm}$. (c) A difference image showing the estimate of the initial pressure distribution reconstructed using time reversal and assuming a uniform density distribution minus the true image. The trapped artifact (the lower circle) is clearly visible. The amplitude of the true initial pressure is underestimated, hence the negative upper circle.

two measurement surfaces (in this case the two points denoted by the dashed lines). When the outgoing wave reaches the density or sound speed change, the impedance mismatch will cause a proportion of the wave to be reflected, as shown in Fig. 1(c) for the sound speed change, and Fig. 1(d) for the density change. Note that on reflection from the density change the pulse undergoes a phase change to become negative. The two pressure time series recorded at the measurement points, $p_{s}(t)$, will thus contain the initial pressure pulse followed by a series of reflections of decreasing amplitude.

If $p_{s}(t)$ is used in a conventional time reversal reconstruction (i.e., as a time-varying Dirichlet boundary condition) with the correct sound speed and density distributions, the initial pressure distribution will be recovered exactly, as the time reversed versions of the reflected waves will recombine with the main pulses correctly. However, if the details of the acoustic heterogeneities are unknown (as will typically be the case in practice) and the mean sound speed and density are used in place of the exact ones, then the recombination will not occur properly and the time reversed versions of the reflected waves-which we have called "vestigial" waves-will remain inside the domain. When they reach the measurement surface, they will be reflected due to the enforced boundary condition and, consequently, trapped within the domain, resulting in artifacts in the final image.

This effect is shown in Fig. 1. The mean, rather than the true, acoustic properties are used in the reconstruction, as indicated by Fig. 1(e). Fig. 1(f) shows the time reversed field at a time corresponding to that in Fig. 1(c). For a perfect time reversal reconstruction these should be identical between the measurement surfaces. (The leftmost wave in Fig. 1(f), beyond the measurement point, is due to the boundary condition being enforced there and is not relevant to the reconstruction. Note the symmetry about the measurement point with its counterpart traveling in the opposite direction.) However, in this case there are clearly several differences. First, the pulse widths are slightly different due to the different sound speeds in the forward and time reversed directions. Second, the negative pulse in Fig. 1(f) is not evident in Fig. 1(c) because, in Fig. 1(c), the wave has yet to reflect from the boundary. As the vestigial waves continue to propagate they will reflect from the measurement surfaces due to the enforced boundary conditions there, and appear as artifacts in the final image, Fig. 1(g). If, as in this case, the boundary condition happens to be being set to zero when the vestigial wave reaches it, it will be reflected with a change of sign. In general, the situation will be more complex, as the boundary condition may be any value. Fig. 1(h) will be discussed in Section IV-B below. In this example, and those given below, a $k$-space propagation model based on (1) was used to simulate the measured data, and a similar model, but with $\rho$ and $c$ set to constant values, was used for the image reconstruction [14], [15].

\section{B. Vestigial Wave Reflection}

In this section, a 2-D example, designed to show clearly the phenomenon of artifact trapping, is described. For this reason, the initial pressure distribution, acoustic heterogeneity, and measurement surface were chosen to be simple geometric shapes, as it is possible in this case to observe the individual vestigial waves being reflecting from the measurement surface by the imposed boundary condition. Fig. 2(a) and (b) shows a density heterogeneity consisting of a step change in the density from 1000 to $700 \mathrm{~kg} / \mathrm{m}^{3}$, and a circular initial pressure distribution with a diameter of $0.5 \mathrm{~mm}$. The square measurement surface is also shown on both figures as a solid black line. Starting at time $t=0$, a circular photoacoustic wave propagates outwards from the initial pressure distribution. When it reaches the density heterogeneity, a scattered wave is reflected back upwards (negative $z$-direction). The acoustic pressure time series are recorded at every pixel forming the measurement surface for times $t=0$ to $t=T$, which in this example is $T=9.42 \mu \mathrm{s}$. In principle, for time reversal imaging it is necessary to record the pressure at the boundary until the pressure in $\Omega$ is zero. In 2-D, this will never (quite) be the case, but in practice it is only necessary to record until the pressure has dropped to a sufficiently low nonzero value. These forward simulations were performed using the k-space method described in [14] and [15], on a $256 \times 256$ pixel square grid, with a sound speed of $1500 \mathrm{~m} / \mathrm{s}$, and time steps of $7.8 \mathrm{~ns}$. Random noise was added at $5 \%$ of the maximum value of the "measured" data, and the measurements were interpolated onto a $304 \times 304$ pixel grid for use in the image reconstruction. (This arrangement was used for all the 2-D simulations.) 

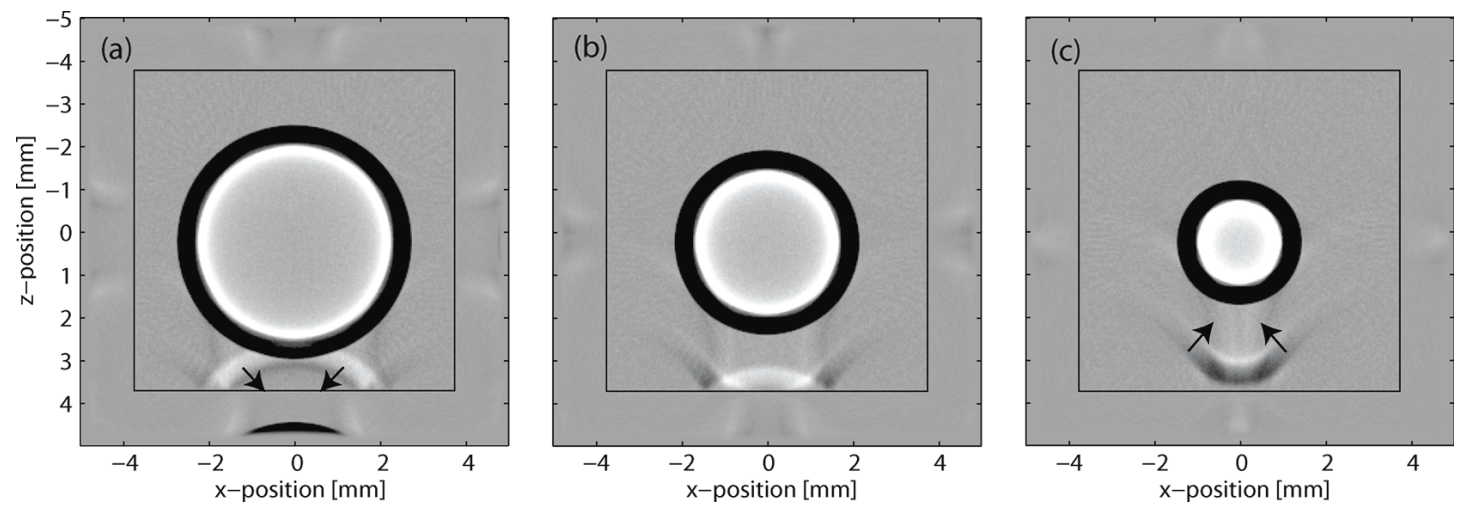

Fig. 3. Artifact trapping, 2-D example: the vestigial wave-the time reversed version of the wave scattered from the density change in Fig. 2(a)—is seen below the main converging circular wavefront, reflecting from the measurement surface because of the boundary condition imposed there by the time reversal imaging algorithm, and converging on its way to becoming an artifact. The difference image, Fig. 2(c), shows the artifact clearly. The snapshots of the wavefield are taken at values of the reverse time variable $\check{t}$ of (a) $T-1.63$, (b) $T-1.24$, and (c) $T-0.77 \mu \mathrm{s}$, where $T$, the duration of the measurements, was $9.42 \mu \mathrm{s}$. The arrows indicate the direction of the vestigial wave. The colorscale was set to enable the vestigial waves to be seen clearly, which resulted in clipping the amplitude of the main circular wavefront.

For the time reversal image reconstruction, the simulated measurements were used as a time-varying Dirichlet boundary condition with the reversed time variable $\breve{t}$ running from 0 to $T$ (equivalent to $t$ from $T$ to 0 ). The wavefield at times $\check{t}=T-1.63, T-1.24$, and $T-0.77 \mu \mathrm{s}$ during the time reversal is shown in Fig. 3. The converging circular wave in the center of the three images (a), (b), and (c) is the time reversed wave that will form the image of the initial pressure distribution. The wave just beneath this is a vestigial wave. In (a), it is traveling downwards towards the measurement surface (black line) on which a boundary condition is imposed. In (b) it has partly been reflected from the surface, and in (c) it is converging to form the artifact shown clearly in Fig. 2(c), which shows the difference between the true and reconstruction initial pressure distributions. It is this reflection of the wave from the measurement surface, that we are referring to as artifact trapping. (In Fig. 3 the color scale has been set so that the lower amplitude vestigial wave is clearly visible.)

It is interesting, although perhaps not surprising, that in this case the amplitude of the distinct artifact [the lower circle in Fig. 2(c)] is very similar to the reduction in the amplitude of the original pressure [given by the upper circle in 2(c)]. Of course, this is just a tutorial example. In general, the vestigial wave will not be so clearly distinguishable from the other waves propagating in $\check{\Omega}$, different shaped measurement surfaces will lead to different shaped final artifacts, the acoustic heterogeneities will be less geometric in shape, and there will be heterogeneities in sound speed as well as density. This simple scenario was arranged merely to illustrate the phenomenon clearly, not represent a realistic case study. The phenomenon itself is, however, a general one and not dependent on the specifics of this particular example.

\section{Comparison to Spherical Mean Algorithm}

Finch et al. [16] have published a PAT reconstruction algorithm based on the inversion of spherical means that is exact in the 2-D homogeneous case, and which is applicable when the measurement surface is circular. For the example above, a square measurement surface was chosen so that the reflection of the vestigial wave could be clearly seen. A similar example is used here, but using a circular sensor surface (inscribed inside the position of the square sensor). The sensor was chosen to have 360 equally spaced pixel-sized elements. For this time reversal imaging, a continuous measurement surface was used, which was divided into 360 equal arcs and the data from each of the 360 sensor elements associated with its corresponding arc. In this way the surface's continuity is maintained [15].

Fig. 4(b) shows the image reconstructed using conventional time reversal. A curved artifact is clearly visible near the bottom of the image. This is different from the disc artifact in Fig. 2(c) because the shape of the measurement surface is different, even though everything else about the two examples is the same. The same artifact is not so clearly visible in Fig. 4(a), which shows the solution obtained using the algorithm in Finch et al. [16]. This is one example of how, in some circumstances, time reversal image reconstruction can result in images with a greater number of artifacts than other reconstruction approaches.

One reason for the superior performance of the Finch $e t$ al. algorithm may be due to the fact that it only uses data from $t=0$ to $t=2 R_{0} / c$, where $R_{0}$ is the radius of the circular measurement surface, so none of the waves scattered and reflected by the heterogeneities that arrive at the sensor surface after $2 R_{0} / c$ are included in the reconstruction process. In contrast, the requirement for the pressure field to be (virtually) zero inside $\Omega$ in the time reversal case, means that much longer time series were used in the reconstruction. The fact that the Finch et al. reconstruction only needs data up to the time when all the wavefront edges - the abrupt changes-have arrived at the sensor in order to reconstruct an exact image, is interesting. It does not need the "tail" of the acoustic waves suggesting they do not carry any extra information even though-because this is in 2-D-they are always nonzero. The advantage of applying this sort of truncation to the data in the time reversal case will be explored below in Section IV-A.

\section{Mitigating ARTifact TRAPPING}

The reconstruction algorithm of Finch et al. proved superior to time reversal, in the example considered above, in terms 

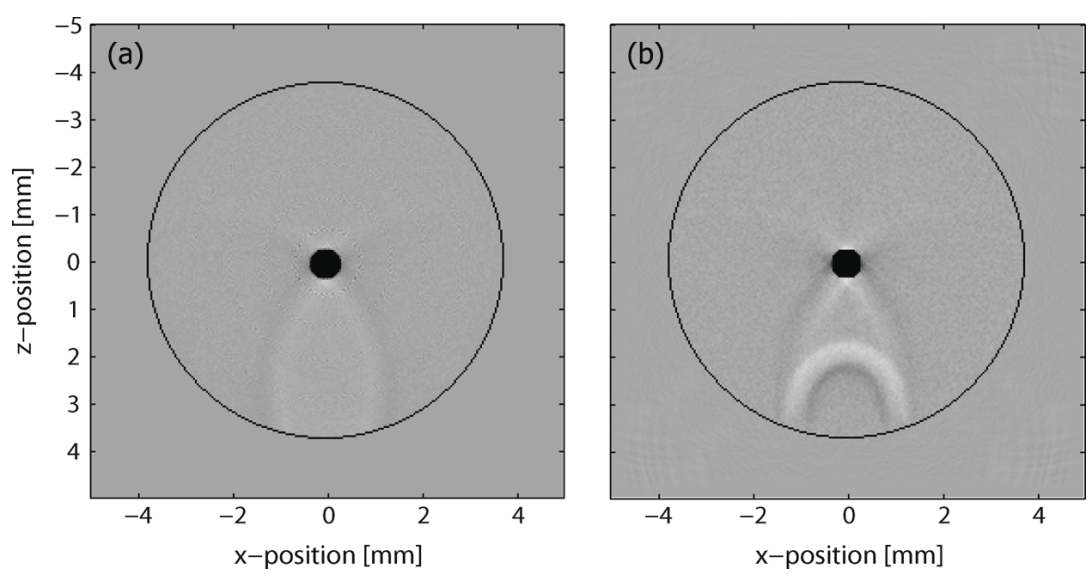

Fig. 4. Artifact trapping, 2-D example: Images reconstructed using data recorded over a circular measurement surface of radius $3.7 \mathrm{~mm}$, with 360 equally spaced sensor elements, for the disc-shaped initial pressure distribution shown in Fig. 2(b). (a) Reconstruction using Finch et al's 2-D algorithm [16] and (b) conventional time reversal image reconstruction. The grayscale is set to $[-0.05,0.05]$ in order to show the artifacts clearly. The curved artifact trapped by the time reversal algorithm is clearly visible in (b) but not in (a).

of the level of artifacts in the image resulting from unknown acoustic heterogeneities. Time reversal, however, remains much more general in its applicability (e.g., Finch et al. require a circular measurement surface) and is considerably faster to compute. (In Matlab, running on a $3 \mathrm{GHz} \mathrm{CPU}$, the Finch algorithm took more than $600 \mathrm{~s}$ compared to $20 \mathrm{~s}$ for the time reversal, even though many of the calculations for the former were performed on a nVidia Quadro FX 3700 GPU using the Matlab GPUmat toolbox (available from gp-you.org). For 3-D reconstructions this $30 \times$ difference in speed will be much greater.) Therefore, because of its speed, versatility and generality, time reversal image reconstruction remains an attractive option, despite its tendency to trap artifacts. In an attempt to improve the performance of time reversal to these sorts of data errors, two ways to reduce the effect of artifact trapping are described below: data truncation and a thresholded boundary condition.

\section{A. Truncated Data}

In three-dimensions, in an acoustically homogeneous medium with sound speed $c_{0}$, a spherical sensor surface with radius $R_{0}$ will detect no photoacoustic signal after time $t_{R}=2 R_{0} / c_{0}$ (Huygens principle). Any waves arriving after time $t_{R}$ cannot be primary photoacoustically generated waves but must be reflections or scattered waves from acoustic heterogeneities. These waves can lead to artifacts in the reconstructions, as described previously, so not including them in the data for the reconstruction seems sensible. In other words, this "range condition" can be used as a justification for truncating the signal at $t_{R}$.

Fig. 5 shows the results from the 3-D simulation equivalent to the 2-D example in Fig. 4. The initial pressure distribution is a ball with diameter $0.5 \mathrm{~mm}$, centered at the origin, the measurement surface is spherical with radius $3.7 \mathrm{~mm}$, also centered at the origin. There is a step change in the density from 1000 to $700 \mathrm{~kg} / \mathrm{m}^{3}$ on the plane $y=-2.8 \mathrm{~mm}$, which gives rise to an artifact when it is not included in the reconstruction. The simulation was performed on a $128 \times 128 \times 128$ grid, and $5 \%$ noise was added to the data. Fig. 5(a)-(c) shows orthogonal slices through the center of the image obtained by time reversing data recorded up to $11.6 \mu \mathrm{s}$. (The initial pressure distribution was of unit amplitude. The grayscale on these images covers the range $[-0.05,0.05]$ in order to show the artifact more clearly.) A curved artifact due to the trapping of a vestigial wave is visible. Fig. 5(d)-(f) shows the same as (a)-(c), except that this time only the data up to $5 \mu \mathrm{s}$ was used. The artifact has been reduced, showing that truncating the data can be beneficial in 3-D.

Unlike propagation in 3-D, propagation in 2-D does not satisfy Huygens principle, as the tails of the photoacoustically generated waves approach zero only in the limit of infinite time. (Although in practice, the tail always falls below the noise floor rather sooner.) In 2-D, then, truncating the data to time $t_{R}$ is not a range condition, as the measured signals are not expected to fall to zero after this time, even in homogeneous media. However, Finch et al.s exact 2-D imaging algorithm, used in Section III-C, uses the data only up to time $t_{R}$, suggesting that the tails of the signals do not carry additional information about the image. On this basis, truncated time series were used for the 2-D time reversal image reconstruction described in Section III-C. The image in Fig. 4(b) was reconstructed using time series up to $9.4 \mu \mathrm{s}$. The same data, but truncated to $t_{R}=5 \mu \mathrm{s}$, was used to reconstruct the image shown in Fig. 6(a). Comparing these two images it is clear that truncating the data can be advantageous in 2-D. (Anastasio et al. [4] take the idea of truncating the data to reduce imaging artefacts due to heterogeneities further, and look at the ameliorating effect of using more severe temporal truncations to times of only $t=R_{0} / c$ : "half-time" reconstruction.)

\section{B. Thresholded Boundary Condition}

Fig. 7(a) shows a simulated pressure time series from the example described in Section III-B (shown in Figs. 2 and 3). The reflected wave is clearly visible as a second pulse with a lower amplitude than the first. In time reversal reconstruction, time series such as this are used to define the pressure at the boundary (the measurement surface) as a function of time, i.e., as a time-varying Dirichlet condition. Whenever the pressure is close to zero, a pressure-release boundary condition, equivalent 

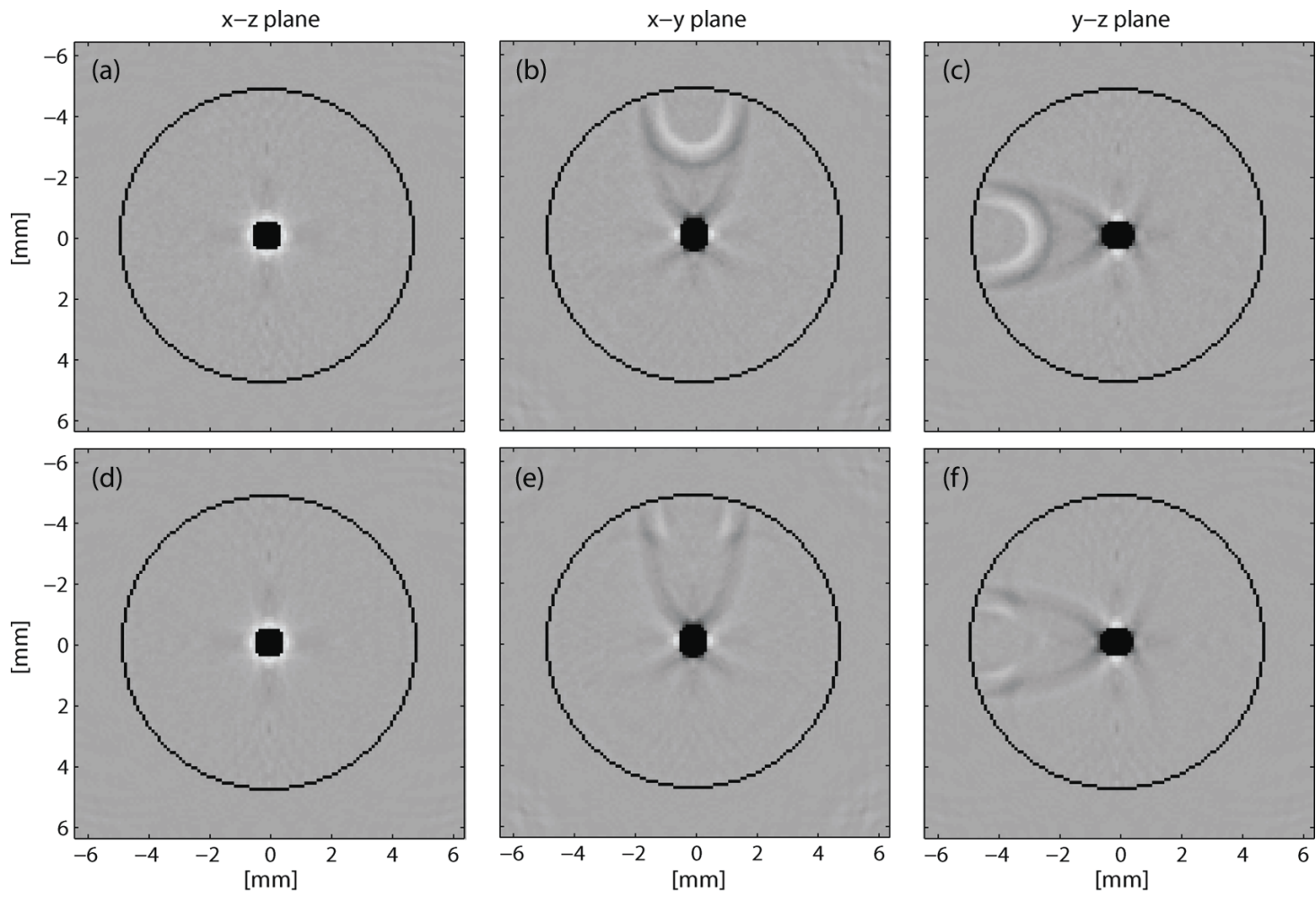

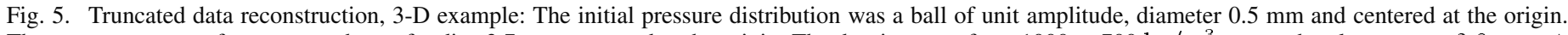

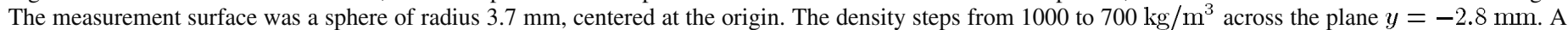

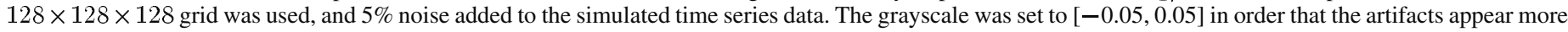

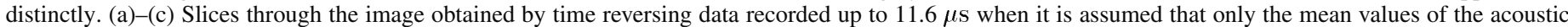

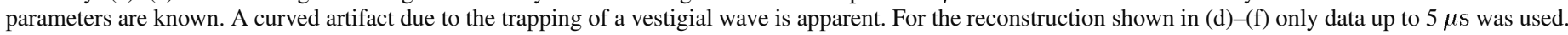
The artifact has been reduced, showing that truncating the data can be beneficial.

in ultrasonics to a water-air interface, will result, which will reflect any incident waves with a reflection coefficient close to -1 . A similar interaction will occur when the boundary is enforced to other values, although the characteristics of the reflected wave will be more complex. It is in this way that the boundary condition-an integral part of the time reversal image reconstruction approach — can act to trap vestigial waves within the image region leading to artifacts in the final image.

Under certain conditions, it may be possible to remove the boundary condition and allow the vestigial waves to pass freely out of the imaging region. Ideally, the boundary condition would be removed whenever and wherever on the boundary such a wave is incident. However, unless the imposed boundary pressure $p_{s}(T-t)$ happens to be zero at that moment, this approach will result in a reduction of the amount of useful data used in the reconstruction (not to mention the difficulty of knowing when the vestigial waves are arriving). A balance must be reached between allowing the vestigial waves to pass across the boundary and ensuring the measured data is fed into the reconstruction. This may be achieved by imposing a thresholded boundary condition of the form

$$
\check{p}\left(x_{s}, \check{t}\right)=p_{s}(T-\check{t}) \text { only if }\left|p_{s}(T-\check{t})\right|>\eta
$$

where $\eta$ is a threshold value. For example, Fig. 7(b) shows the same time series as Fig. 7(a), but zoomed in to show the low amplitude region, and a threshold set at an amplitude of $\pm \eta$.
When $\left|p_{s}\left(x_{s}, T-\check{t}\right)\right| \leq \eta$ (which is computed separately for each position on $\breve{S}$ ), no boundary condition is imposed and the residual waves can freely propagate out of the imaging region. The selection of the numerical value for $\eta$ will be a tradeoff between reducing artifact trapping and including sufficient data in the reconstruction.

One-Dimensional Example: Fig. 1(h) shows the estimate of the initial pressure distribution for the 1-D example described in Section III-A, reconstructed using time reversal with this thresholded boundary condition. It is clear that the vestigial waves are now able to propagate freely through the measurement surface (the two points marked by dashed lines). The reconstruction of the main wave pulse remains almost identical (the coherent interaction between the main wave pulses is barely changed), while the two reflection artifacts are no longer present within the imaging region.

Two-Dimensional Example: For the 2-D case, Fig. 8(a) shows the difference between the true initial pressure distribution [Fig. 2(b)] and its estimate obtained using the thresholded approach. As in Fig. 2(c), the reconstruction assumed the mean value for the density; however, unlike Fig. 2(c) which used conventional time reversal reconstruction, here a boundary condition threshold was set at $\eta=0.003$. Clearly the amplitude of the main artifact is lower, as expected, because the vestigial wave was no longer trapped by the boundary condition. However, the thresholding did result in new, lower level, artifacts in the form of arcs in the vicinity of the source. This exemplifies 

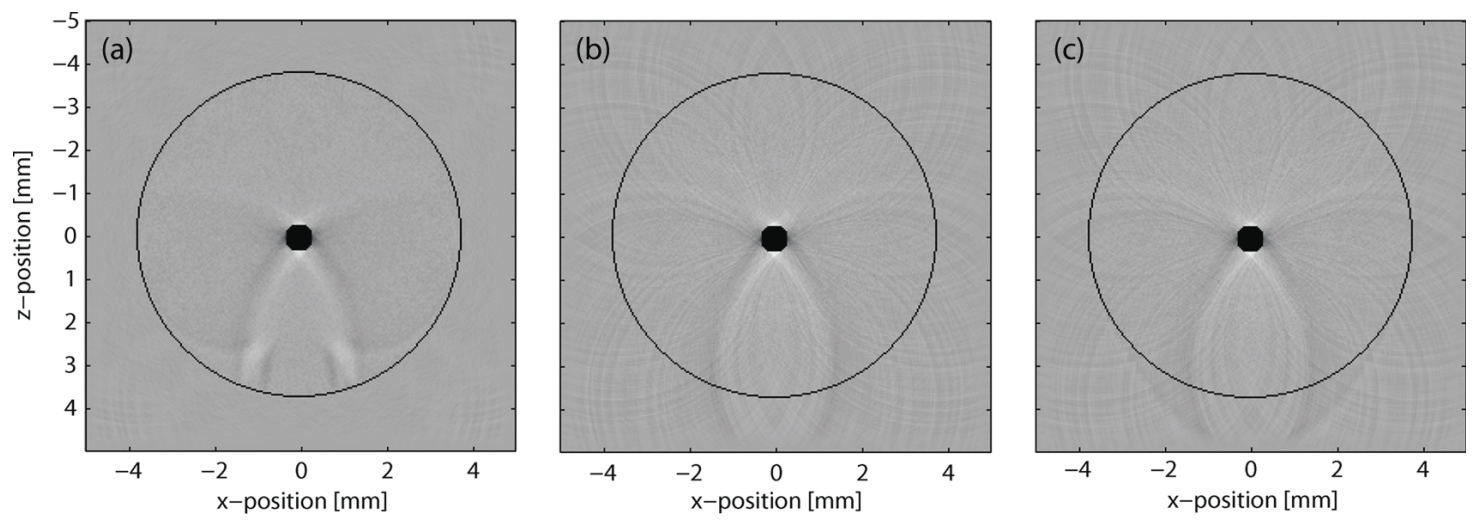

Fig. 6. Truncated and thresholded reconstruction, 2-D example: Images reconstructed using data recorded over a circular measurement surface (as in Fig. 4). (a) Conventional time reversal image reconstruction using only data up to time $t=2 * R_{0} / c$, where $R_{0}$ is the radius of the circular measurement surface and $c$ is the mean sound speed. Comparison with Fig. 4(b) clearly shows that truncating the data can have the effect of removing part of the curved artifact. (b) Thresholded time reversal reconstruction with $\eta=0.005$. The artefact visible in Fig. 4(b) has been removed at the expense of a slight increase in lower level artifacts spread across the image. (c) Truncated and thresholded time reversal reconstruction with $\eta=0.005$. Thresholding reduces the level of the artifact further than truncation alone, showing that the two approaches are complementary and not equivalent. In this case, little advantage is gained by truncating the data as well as thresholding. The grayscale in all the images is set to $[-0.05,0.05]$.

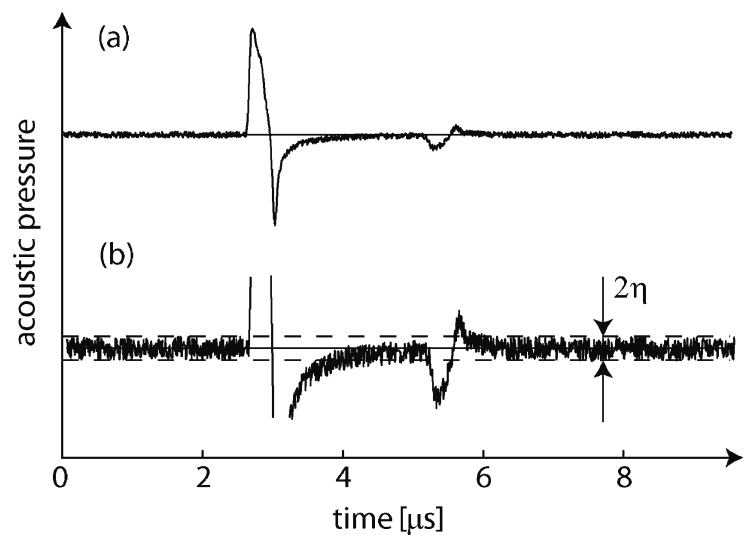

Fig. 7. (a) Simulated (2-D) pressure time series from the example in Figs. 2 and 3 , with the reflected wave clearly visible. In the time reversed reconstruction, the many values close to zero will result, effectively, in a reflective "pressurerelease" boundary wherever it is imposed. In this way, the boundary condition can act to trap vestigial waves within the image region leading to artifacts in the final image. (b) Detail of the time series in (a) close to zero, with a possible threshold condition indicated with values of $\pm \eta$.

the general point, that there will be a tradeoff between reducing the effect of artifact-trapping, and maintaining the amount of information used to reconstruct the image.

Choice of Threshold Parameter $\eta$ : The thresholded time reversal algorithm can provide an infinite family of images: one for each value of the threshold parameter $\eta$. Setting $\eta=0$, for instance, gives an image equivalent to that obtained from conventional time reversal imaging. At the opposite extreme, setting $\eta$ larger than any value of the measured pressure will result in an image that is zero everywhere. It is therefore necessary to strike a balance somewhere between these two extremes. Which of the images - which value of $\eta$-is the correct one to choose? All the possible images will be approximations to the true image, and none of them will be exactly correct, so a criteria to determine the "best" is needed. A useful comparison can be made here to image deblurring and related techniques, in which a regularization parameter must be chosen in a largely analogous way to the problem of selecting $\eta$ here. This problem has been widely discussed in the literature (see, for example, [17].) The optimum value of $\eta$ will depend on the criteria that is used to choose it. It is likely that in practice, the signal-to-noise ratio in the measurements will affect the choice of $\eta$ too. If $\eta$ is set close to the noise floor, as indicated in Fig. 7, then all of the signal above the noise will be included in the reconstruction. This should not be taken as a strict criterion, however, as there maybe useful data masked by the noise that nevertheless combines constructively during the image reconstruction process, while the noise reduces through an averaging effect. The criteria may be qualitative-based on what the images look like, for instance-or more quantitative, using techniques such as the L-curve or generalized cross validation. In the simple case where the true image is known, such as may be the case when testing algorithms with simulated data or using phantoms, an objective measure of error could be used to choose $\eta$, such as the $l^{2}$ norm of the difference between the images. The $l^{2}$ norm (the root mean squared error) was calculated for a range of values of $\eta$ for the example above, and is shown in Fig. 8(b). The smallest error occurs when $\eta=0.003$, the difference image corresponding to which is shown in Fig. 8(a).

Circular Detector Example: In Fig. 6(b), a thresholded algorithm with $\eta=0.005$ has been used, which largely removes the curved artifact visible in Fig. 4(b). Fig. 6(c) shows the image obtained using temporally truncated data with the thresholded algorithm. Thresholding [Fig. 6(b)] reduces the level of the artifact further than truncation alone [Fig. 6(a)] showing that the two approaches are not equivalent. In this particular case, little advantage is gained by truncating the data as well as thresholding [Fig. 6(c)] but this will not necessarily be the case in general, as the two strategies could be complementary.

Random Heterogeneities: As a final example, consider the propagation of a circular wave through a medium with random acoustic heterogeneities, as shown in Fig. 9. Here, the waves resulting from a $0.5 \mathrm{~mm}$ diameter, disc shaped, initial pressure distribution with unit amplitude, and propagating within a medium with random variations in sound speed and density, is recorded over a circular measurement surface ( $3.7 \mathrm{~mm}$ radius). 


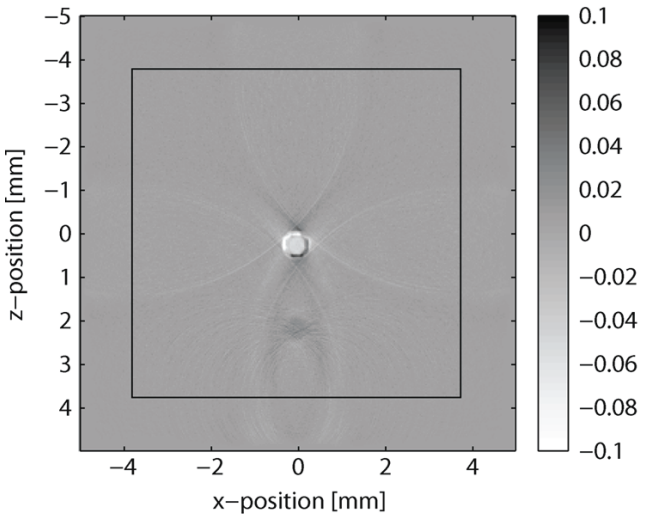

(a)

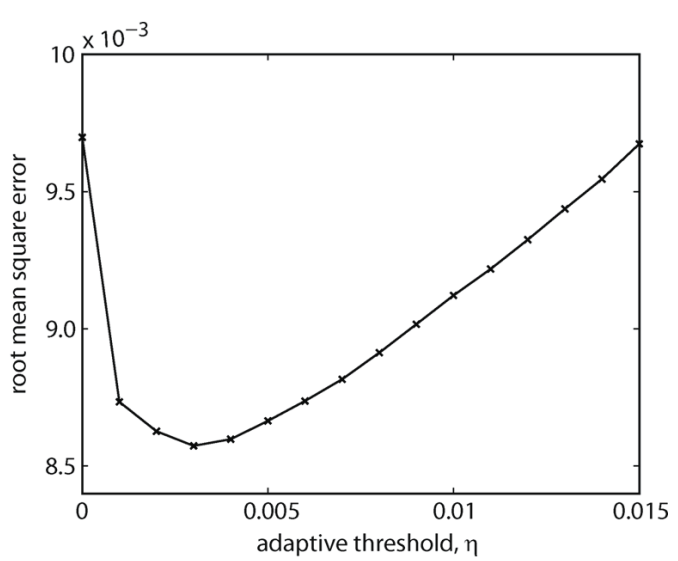

(b)

Fig. 8. Thresholded reconstruction, 2-D example: (a) image of the difference between the true initial pressure distribution, Fig. 2(b), and the estimated reconstruction using time reversal with a thresholded boundary condition and $\eta=0.003$. Comparison with the difference image for the conventional reconstruction, Fig. 2(c), shows that the thresholded boundary approach achieves almost total removal of the main artifact. (b) The root mean squared error in the reconstructed image as a function of the threshold parameter $\eta$.
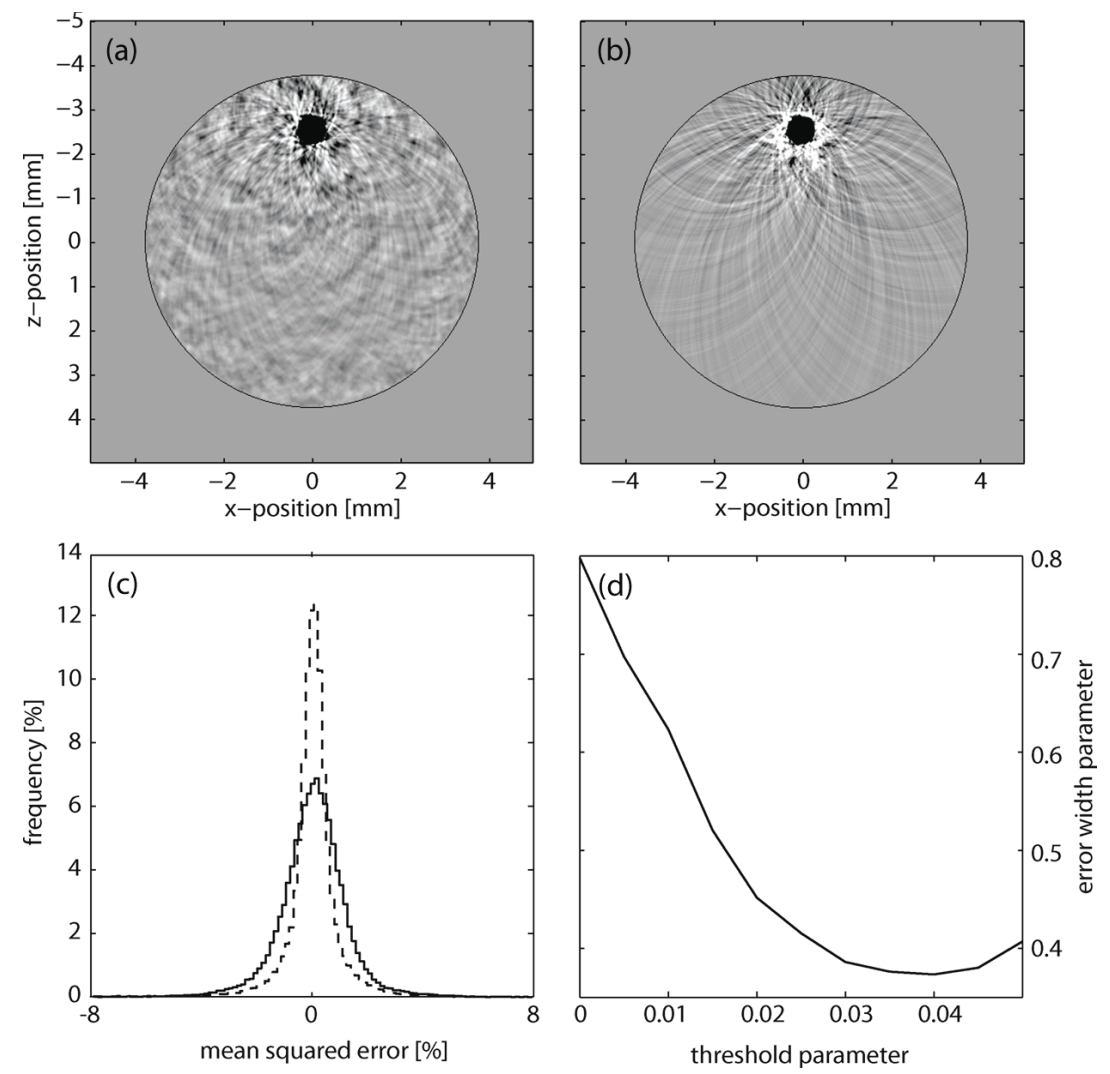

Fig. 9. Artifact trapping with random heterogeneities, 2-D example. (a) Reconstruction using conventional time reversal. (b) Reconstruction using thresholded time reversal with $\eta=0.04$, showing a reduction in the level of artifacts, especially closer to the boundary. (c) Error histograms for conventional and thresholded time reversal. (d) Reduction of the Lorentzian width parameter $\sigma$ (an estimate of the level of error) with the time reversal threshold $\eta$.

The sound speed and density were set by an algorithm that added square sound speed heterogeneities, whose sizes and amplitudes were chosen randomly from Gaussian distributions, to a mean background value in random locations. The sound speed heterogeneity amplitude was modelled as Gaussian with a mean of $1500 \mathrm{~m} / \mathrm{s}$ and a standard deviation of 0.04 . The density was selected as $\rho=0.893 * c-349$, which is a relation derived from the measured properties of soft tissue [18]. The simulations were performed on a $512 \times 512$ grid. While this model may not exactly mimic tissue structure, it provides a similarly heterogeneous medium with which to test the robustness of the algorithms.

The simulated data, truncated at time $t_{R}$, was used in a time reversal reconstruction (that assumed uniform acoustic properties with the value of the mean sound speed and density) with both conventional and thresholded boundary conditions (with $\eta=0.04$ ), shown in Fig. 9(a) and (b), respectively. The grayscale range is $\left[\begin{array}{ll}-0.05 & 0.05\end{array}\right]$. Applying the thresholded boundary condition has reduced the overall level of artifacts, especially near the outer edges of the image. Histograms com- 
paring the magnitude errors in the reconstructions are shown in Fig. 9(c), demonstrating the superiority of the thresholded algorithm when random variations of this sort are present [the solid line corresponds to Fig. 9(a), the dashed line to Fig. 9(b)]. To make a quantitative error comparison, a two-parameter centered Lorentzian $f=A /\left(1+\eta^{2} / \sigma^{2}\right)$ was fitted to these histograms. The reduction of the Lorentzian width parameter $\sigma$, an indicator of the level of error, as the time reversal threshold value $\eta$ is increased is shown in Fig. 9(d). As $\eta$ increases, there is a reduction in $\sigma$ to a minimum at which the value of $\eta$ reaches the noise floor. Further increases in $\eta$, which will decrease the time the boundary condition is enforced, will result in degradation of the image as the amount of data used in the reconstruction is reduced. This example demonstrates that thresholded reconstruction can reduce the overall level of background artifact "noise" in an image, as well as reduce the amplitude of specific artifacts as described above.

\section{AdDitional CONSIDERATIONS}

Acoustic Absorption: None of the models used to simulate the data in the examples above included the effect of acoustic absorption. In practice, absorption will reduce the amplitude of reflections that have travelled some distance through the tissue, but will not fundamentally affect artifact trapping. Scattered waves will still be detected, albeit at lower amplitudes, and will still result in vestigial waves during the time reversal process. Where absorption is significant and quantitatively accurate reconstructions are required, an algorithm that accounts for the absorption (that counteracts it, so wave amplitudes grow as the waves propagate) may be used in the image reconstruction. Such a model, when used for time reversal imaging, rather than improve the image accuracy could exacerbate artifact trapping by increasing the amplitudes of the vestigial waves.

Scattering From Incomplete Measurement Surfaces: Often, for practical reasons, it is necessary to use an incomplete measurement surface. The surface could be truncated, for instance, or measurements made at only a number of sampling points totaling only a fraction of the whole surface. When time reversal is used without a complete surface, it is necessarily an approximation, although it is interesting that it can still often construct good approximate images in these cases [15]. When the measurements are made at discrete points, the time reversal algorithm fixes the pressure at these points which can make them act as scatterers. In this case, rather than trapping a vestigial wave whole, as it were, it may scatter it back into the imaging region. This can result in arc-like artifacts across the image. One way to reduce these artifacts is to time-reverse with a complete measurement surface acting as the boundary, and interpolate the measured data onto it [15].

\section{Summary AND CONCLUSION}

The phenomenon of artifact trapping in time reversal photoacoustic image reconstruction was identified and described. In time reversal image reconstruction, when a model of propagation in an acoustically homogeneous medium is used to time reverse measurements made in a heterogeneous medium, vestigial waves appear in the image domain. These can subsequently become trapped by the enforced boundary condition inherent in time reversal imaging and appear as artifacts in the final image. Truncating the measurement data and using an adaptive thresholded boundary condition were both shown to mitigate this source of error.

\section{REFERENCES}

[1] M. Xu and L. V. Wang, "Photoacoustic imaging in biomedicine," Rev. Sci. Instrum., vol. 77, no. 4, pp. 041101-041122, 2006.

[2] Photoacoustic Imaging and Spectroscopy, L. V. Wang, Ed. Boca Raton, FL: CRC, 2009.

[3] Y. Xu, L. V. Wang, G. Ambartsoumian, and P. Kuchment, "Reconstructions in limited-view thermoacoustic tomography," Med. Phys. vol. 31, no. 4, pp. 724-733, 2004.

[4] M. A. Anastasio, J. Zhang, X. Pan, Y. Zou, G. Ku, and L. V. Wang, "Half-time image reconstruction in thermoacoustic tomography," IEEE Trans. Med. Imag., vol. 24, no. 2, pp. 199-210, 2005.

[5] P. Kuchment and L. Kunyansky, "Mathematics of thermoacoustic tomography," Eur. J. Appl. Math., vol. 19, no. 2, pp. 191-224, 2008.

[6] Y. Hristova, P. Kuchment, and L. V. Nguyen, "Reconstruction and time reversal in thermoacoustic tomography in acoustically homogeneous and inhomogeneous media," Inv. Probl., vol. 24, no. 5, p. 055006, 2008.

[7] D. R. Jackson and D. R. Dowling, "Phase conjugation in underwater acoustics," J. Acoust. Soc. Am., vol. 89, no. 1, pp. 171-181, 1991.

[8] M. Fink, "Time reversal of ultrasonic fields. I. Basic principles," IEEE Trans. Ultrason., Ferroelectr., Freq. Control., vol. 39, no. 5, pp. 555-566, 1992.

[9] M. Fink, "Time-reversal acoustics in complex environments," Geophys., vol. 71, no. 4, pp. SI151-SI164, 2006.

[10] E. Bossy, K. Daoudi, A. C. Boccara, M. Tanter, J. F. Aubry, G Montaldo, and M. Fink, "Time reversal of photoacoustic waves," Appl. Phys. Lett., vol. 89, p. 184108, 2006.

[11] Y. Xu and L. V. Wang, "Time reversal and its application to tomography with diffracting sources," Phys. Rev. Lett., vol. 92, no. 3, p. 033902, 2004.

[12] P. Burgholzer, G. J. Matt, M. Haltmeier, and G. Paltauf, "Exact and approximative imaging methods for photoacoustic tomography using an arbitrary detection surface," Phys. Rev. E, vol. 75, no. 4, p. 046706 , 2007.

[13] Y. Hristova, "Time reversal in thermoacoustic tomography-An error estimate," Inv. Probl., vol. 25, p. 055008, 2009.

[14] B. T. Cox, S. Kara, S. R. Arridge, and P. C. Beard, "k-space propagation models for acoustically heterogeneous media: Application to biomedical photoacoustics," J. Acoust. Soc. Am., vol. 121, no. 6, pp. 3453-3464, 2007.

[15] B. E. Treeby and B. T. Cox, "k-Wave: A MATLAB toolbox for the simulation and reconstruction of photoacoustic wave-fields," J. Biomed. Opt..

[16] D. Finch, M. Haltmeier, and Rakesh, "Inversion of spherical means and the wave equation in even dimensions," SIAM J. Appl. Math., vol. 68 , no. 2, pp. 392-412, 2007.

[17] M. Bertero and P. Boccacci, Introduction to Inverse Problems in Imaging. London, U.K.: Inst. Physics, 1998.

[18] T. D. Mast, "Empirical relationships between acoustic parameters in human soft tissues," Acoust. Res. Lett., vol. 1, no. 2, pp. 37-42, 2000. 